OPEN ACCESS

Edited by:

Zong Wei,

Mayo Clinic Arizona, United States

Reviewed by:

Liu Wang,

Mayo Clinic Arizona, United States Mohammed Mahboobuddin Ahmed, King Saud University Medical City,

Saudi Arabia

*Correspondence:

Eli Ipp

ipp@/undquist.org

Specialty section:

This article was submitted to Diabetes: Molecular Mechanisms, a section of the journal

Frontiers in Endocrinology

Received: 01 July 2021 Accepted: 28 September 2021

Published: 29 November 2021

Citation:

Ipp E (2021) Diabetic Retinopathy and Insulin Insufficiency: Beta Cell Replacement as a Strategy to Prevent Blindness.

Front. Endocrinol. 12:734360. doi: $10.3389 /$ fendo.2021.734360

\section{Diabetic Retinopathy and Insulin Insufficiency: Beta Cell Replacement as a Strategy to Prevent Blindness}

\author{
Eli Ipp*
}

Department of Medicine, The Lundquist Institute at Harbor-University of California Los Angeles (UCLA) Medical Center, Torrance, CA, United States

Diabetic retinopathy (DR) is a potentially devastating complication of diabetes because it puts patients at risk of blindness. Diabetes is a common cause of blindness in the U.S. and worldwide and is dramatically increasing in global prevalence. Thus new approaches are needed to prevent this dreaded complication. There is extensive data that indicates beta cell secretory failure is a risk factor for DR, independent of its influence on glycemic control. This perspective article will provide evidence for insufficient endogenous insulin secretion as an important factor in the development of DR. The areas of evidence discussed are: (a) Presence of insulin receptors in the retina, (b) Clinical studies that show an association of beta cell insufficiency with DR, (c) Treatment with insulin in type 2 diabetes, a marker for endogenous insulin deficiency, is an independent risk factor for DR, (d) Recent clinical studies that link DR with an insulin deficient form of type 2 diabetes, and (e) Beta cell replacement studies that demonstrate endogenous insulin prevents progression of DR. The cumulative data drive our conclusion that beta cell replacement will have an important role in preventing DR and/or mitigating its severity in both type 1 diabetes and insulinopenic type 2 diabetes.

Keywords: diabetes, retinopathy, beta cell (B-cell), insulin secretion, beta cell failure

\section{INTRODUCTION}

Diabetic retinopathy (DR) is a devastating complication of diabetes because it can lead to visual impairment and blindness. Diabetes is the most common cause of blindness in the working age population of the U.S. As prevalence of diabetes increases worldwide, new approaches are needed to prevent blindness. Pathogenesis of DR is multifactorial, but there is increasing evidence that diminished beta cell function is an independent risk factor for DR. This perspective article will put forth evidence for the role of insufficient endogenous insulin as an important factor in the development of DR that may therefore be amenable to prevention by beta cell replacement.

There are five areas of evidence that will be discussed (Figure 1):

(a) Evidence for, and role of, insulin receptors in the retina.

(b) Clinical data that demonstrate an association of beta cell insufficiency with the presence and severity of DR.

(c) Therapeutic use of insulin in type 2 diabetes as a marker for insulin deficiency and thus as an independent risk factor for DR. 
Evidence that Supports Role for Beta Cell Replacement to Prevent Progression of Diabetic Retinopathy

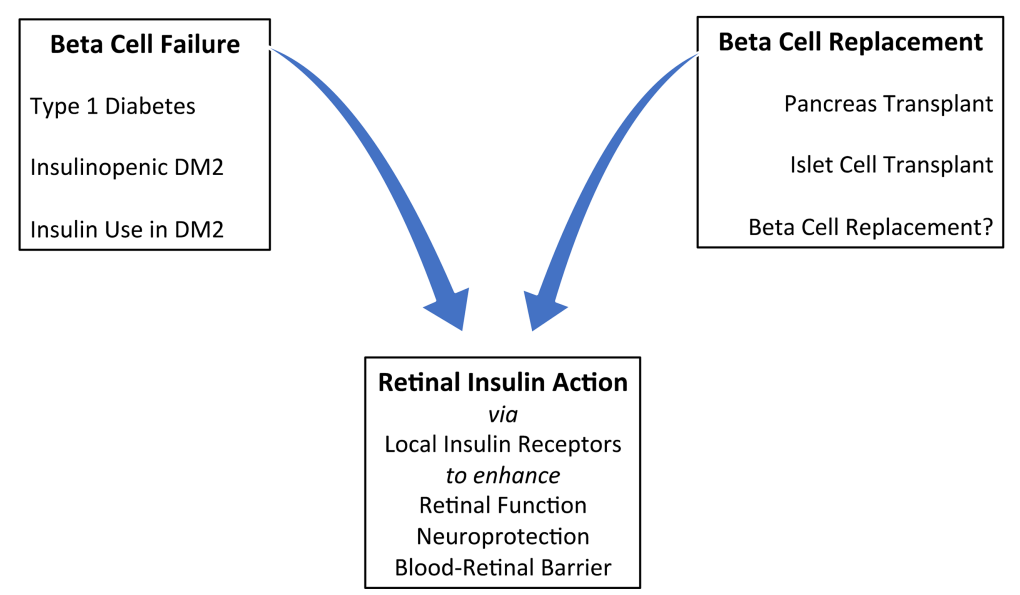

FIGURE 1 | Evidence that Supports a Role for Beta Cell Replacement to Prevent Progression of Diabetic Retinopathy: insulin deficiency in the role of risk factors, effects of replacement methods, and retinal effects of insulin. DM2 = Type 2 Diabetes.

(d) Recent prospective clinical studies linking development of DR with a newly described subtype of type 2 diabetes, that is characterized by insulin insufficiency.

(e) Pancreas and islet transplantation as a source of endogenous insulin and C-peptide that have a beneficial effect on DR.

The accumulation of data that derives from these disparate areas of investigation drives our conclusion that beta cell replacement will have an important role in preventing DR and/or mitigating its severity in both type 1 and insulinopenic type 2 diabetes.

\section{Insulin Receptors in the Retina}

In order to consider a direct link of insulin secretion to DR that does not involve other biological pathways, evidence for insulin action in the retina is essential. The presence of retinal insulin receptors is thus an important pre-requisite. Though the retina is not regarded as a typical end organ for insulin action such as muscle, fat and liver - precedent exists for insulin receptors in tissues that are not classical insulin targets. The retina is indeed a non-classical target for insulin action, as evidenced by the finding that insulin receptors are widely expressed in the human retina (1). Insulin receptors have been localized to the nerve fiber layer, ganglion cells, Mller glia, outer nuclear layer, inner segments of rods and cones, the outer limiting membrane and retinal pigment epithelium (RPE) (1). Also, insulin signaling within the RPE occurs in dose response fashion (2).

DR has traditionally been defined by vascular changes in the retina, which form the basis for grading and classification of DR (3). It is now thought that neural involvement is an equally important participant in the pathogenesis of DR (4). It is therefore relevant that insulin has been proposed as an important neuroprotective factor (5-16), potentially protecting against the development and progression of DR. Insulin receptors are widely expressed in the neural retina (1). In rats with streptozotocin-induced diabetes, insulin has been reported to protect retinal function, reducing retinal cell apoptosis, glial activation, VEGF upregulation, and Brain-Retinal-Barrier damage (5). Insulin receptors expressed by the RPE appear to provide support to photoreceptors in the diabetic retina (6). Recent data also suggest that, even more than insulin, the pro-hormone proinsulin may exert significant neuroprotective actions in the retina (7).

In addition to the finding of receptors, insulin mediated intracellular pathways have been shown to play a role as prosurvival factors in the retina, acting through phosphoinositide-3kinase (PI3K), protein-kinase B (Akt) and the ribosomal protein S6 kinase, p70S6K, to protect the retina from apoptosis or stress; including damage due to light (8-10). Loss of the insulin receptor in rod photoreceptors leads to diminished PI3K and Akt signaling and increased sensitivity to light induced photoreceptor degeneration (11). Insulin has also been shown to be necessary for photoreceptor survival and activity (11-15). The insulin receptor-PI3K signaling pathway provides neuroprotection to cones (16). Loss of PI3K in cones triggers cone degeneration not protected by rod-derived cone survival factors. It has therefore been suggested that the insulin receptor-PI3K signaling pathway may be a target for neuroprotective therapeutic intervention (16).

Thus, accumulating evidence supports both the presence of insulin receptors in multiple sites in the retina as well as a role for insulin mediated pathways in maintaining normal physiological function. Though more work needs to be done in this area, it appears that a basic cellular framework exists for insulin action in the retina including a likely neuroprotective role.

\section{Clinical Studies}

Glycemic control is a key risk factor in determining the development of DR in type 1 and type 2 diabetes $(17,18)$, 
and this information has driven the adoption of glycemic goals as standards of care for diabetes management, at HbA1c levels of $7 \%$. Though key to the development and progression of DR, the mechanisms by which glycemia influences the development of DR have been questioned (19). Also, glycemic control as a management strategy may have its limitations in DR prevention, as shown in studies of type 2 diabetes in which further improvement in $\mathrm{HbA} 1 \mathrm{c}$ below $7 \%$ did not provide additional benefit to reduce $\operatorname{DR}(20,21)$. DR progressed in these studies, independent of improved glycemic control. This strongly suggests that factors other than glycemic control may be involved in the progression of DR.

In type 1 diabetes, residual endogenous insulin secretion was first shown to protect patients against development of DR (22). In an early follow-up (6 years) of the Diabetes Control and Complications Trial (DCCT) beta cell function, measured as residual C-peptide, showed a clear protective role in DR. At that stage of the trial, there was a sufficient number of participants with some residual C-peptide secretion (56\%) to demonstrate this protective effect (22). But there are limits to this protection. In long-standing type 1 diabetes when beta cell function is so depleted, protection against DR no longer occurs (23). In the latter report, after 37 years of DCCT and its follow up study (DCCT/EDIC), only 12\% had residual C-peptide responses, providing an explanation for the lack of a demonstrated beneficial effect on DR.

Importantly, in type 1 diabetes glycemic control was shown to be irrelevant to protection provided by endogenous beta cell secretion - in the 6 year DCCT trial, an effect of residual Cpeptide to protect the retina was found, irrespective of the $\mathrm{A} 1 \mathrm{C}$ achieved in each of the experimental arms (22). In other words, even in the 'protected' group in good glycemic control of A1C about $7 \%$, the development of DR was significantly dependent on residual C-peptide. This suggests that in addition to the effects of glycemic control, other factors are also relevant - specifically, residual beta cell function.

In type 2 diabetes, a clear relationship between residual insulin and DR was initially more difficult to show. Some studies demonstrated that lower levels of $\mathrm{C}$ peptide are associated with DR (24-27), while other studies did not (28-30). The GOLDR (Genetics of Latino Diabetic Retinopathy) cohort (31) was an opportunity to resolve this question by introducing three distinct methodological advantages: a cohort large enough to include analyzable subgroups, measurement of circulating insulin in addition to C-peptide, and statistical approaches to deal with specific confounders. This study confirmed an inverse correlation of DR with $C$ peptide and insulin in type 2 diabetes (31).

The GOLDR study (31) differed from previous studies in that it examined the relationship of $\mathrm{C}$-peptide and insulin within the entire spectrum of DR severity and used 7-field digital imaging with standard ETDRS grading criteria. This was the first study to measure insulin as well as C-peptide concentrations, to better represent residual beta-cell function. It was also the first study to evaluate all categories of DR severity and residual beta cell function. The deficit in endogenous insulin secretion was associated with increasingly severe DR. Plasma C peptide concentrations paralleled plasma insulin, suggesting that insulin concentrations reflect insulin secretion- and therefore beta-cell function. This association of circulating insulin and $\mathrm{C}$ peptide with the presence and severity of DR remained highly significant after adjusting for well-known risk factors for DR, including diabetes duration and notably, was independent of glycemic control (31). A concurring earlier study found lower post-prandial insulin associated with DR (32).

Insulin secretory failure is a complex outcome to evaluate in clinical studies of diabetic complications, such as DR. Circulating insulin reflects rates of secretion and clearance. Renal clearance is the main mechanism for $\mathrm{C}$ peptide degradation, while insulin is also cleared by the liver. The more severe DR, the more likely renal clearance will be impaired and therefore that insulin and Cpeptide concentrations will be increased, no longer reflecting accurately rate of insulin secretion. By appropriate statistical analysis, Kuo et al. confirmed that worse DR occurred with decreasing $\mathrm{C}$ peptide, reinforcing the importance of deficient beta-cell function in DR (31).

It is therefore likely that discrepant results in earlier type 2 diabetes studies, all of which reported $C$ peptide/DR relationships without insulin measurements, may be due to the complexity of using $\mathrm{C}$ peptide as a marker of beta-cell function in the presence of kidney disease.

The results of the GOLDR study suggest that besides attention to glycemic control, maintenance of beta-cell function or betacell mass in type 2 diabetes may also be justified as a therapeutic goal, thus warranting further investigation (31).

\section{Therapeutic Insulin Use as a Risk Factor for DR in Type 2 Diabetes}

Multiple studies of type 2 diabetes have shown that use of insulin to manage glycemic control is an independent risk factor for DR (33-35). Tudor (33) in the San Luis Valley Diabetes Study, Colorado found an odds ratio for any DR (95\% CI) $=8.45$ (2.65-26.97), in patients on insulin vs. no medications. Jones (34) in the Norfolk Diabetic Retinopathy Study showed that compared to diet control only, the adjusted hazard ratio (95\% $\mathrm{CI})=2.17(1.68-2.81)$ if participants were using insulin. Thomas (35) compared insulin use to diet control with an adjusted hazard ratio $=2.03(1.89-2.18)$ for $\mathrm{DR}$.

What could account for this relationship between use of insulin and the increased likelihood of developing DR? The reflex conclusion has been that insulin use signals poor glycemic control, and while this is generally true, it has led to overlooking the possibility that insulin use reflects beta cell failure and that lack of endogenous insulin might have a direct influence in DR pathogenesis. Impairment of beta cell function is integral to the pathogenesis of type 2 diabetes, but most patients nevertheless do not require insulin treatment to control blood glucose. Only those with more severe beta cell decompensation require exogenous insulin to manage their diabetes, and so insulin treatment becomes a de facto marker for endogenous insulin deficiency in the face of the insulin resistance in type 2 diabetes (36). 
Modern strategies to prevent diabetic blindness focus on risk factors that are amenable to change, such as glycemic and hypertension control (37). Future approaches to prevent blindness will also use large data to derive DR risk stratification protocols to identify specific patients at high risk to target for risk factor amelioration. Insulin use per se is not usually targeted because the assumption is that it is a marker for poor glycemic control rather than a marker for endogenous insulin deficiency in the pathogenesis of DR. This perspective article argues for endogenous insulin deficiency to be recognized in its own right as a direct risk factor independent of glucose control. In this setting, replacement using beta cells can be seen as a logical strategy once methods are developed that simplify the methodology and make it available for more widespread use, such as immune evasion and use of insulin secreting islet organoids (38).

\section{New Prospective Clinical Studies Identifying an Insulinopenic Sub-Type in Type 2 Diabetes}

Type 2 diabetes has long been considered a heterogeneous disease (36). The classical phenotype of an obese middle-aged person with insulin resistance, hypertension, dyslipidemia and increased waist circumference does not include all patients with type 2 diabetes, some of whom are older, leaner, or more insulin sensitive (39). In 2018, Ahlqvist introduced a novel approach to classification of diabetes using cluster analysis. These investigators identified four different clusters of type 2 diabetes, with an additional autoimmune cluster defined by positive antiGAD antibodies (39). Most notably, a type 2 cluster identified as an insulin deficient group was also the most likely cluster to develop DR. Thus, this clinical study demonstrated rather conclusively in prospective fashion that belonging to an endogenous insulin deficient group was a risk factor for DR. Another (insulin resistant) cluster were more likely to develop diabetic kidney disease.

These type 2 diabetes clusters, defined at diabetes onset seem to remain consistent even after several years of diabetes, suggesting that the degree of homogeneity within clusters might reflect different etiologies or pathogeneses that remained true despite the passage of time (40). These clusters were confirmed in other populations in China (41) and the U.S. (41). In a retrospective Japanese study, cluster analysis revealed an association between an insulin deficient cluster and DR (42). In Asian Indians, a cross-sectional study of about 20,000 patients with type 2 diabetes showed a similar relationship with DR and the insulin deficient cluster (43).

Of importance for the view presented in this perspective, is the identification of the insulin deficient cluster, and that it was associated with a higher incidence of $\operatorname{DR}(40,42,43)$. This provides powerful new information illustrating that in type 2 diabetes, endogenous insulin and/or C-peptide (44) secretion, when it fails, may be responsible for development or progression of DR.

In another large prospective trial (Veterans Affairs Diabetes Trial - VADT) of the effect of tight glycemic control on DR, no benefit was found with better A1C in the intervention arm (45). Despite this, in the entire cohort higher baseline C-peptide concentrations were protective for both incidence and progression of DR (45). DR incidence was reduced by $67.2 \%$, and DR progression was reduced $47 \%$ for each $1 \mathrm{pmol} / \mathrm{ml}$ increase in baseline C-peptide, respectively, another indication that residual insulin secretion may influence DR independent of glycemic control.

\section{Results of Pancreas and Islet Transplantation as a Source of Endogenous Insulin and C-Peptide on DR}

If endogenous insulin deficiency contributes to DR, can replacement of beta cells reverse this course? If endogenous insulin is more effective in preventing DR progression than the exogenous form, a positive effect upon DR after beta cell replacement should be found. Indeed, data derived from clinical transplant studies in type 1 diabetes support a protective role in DR for endogenous insulin secretion.

In a recent meta-analysis (46) islet cell transplantation was associated with diminished DR progression compared with either intensive or standard medical therapy $(5.2 \%$ vs $25.0 \%$; relative risk $=0.25 ; 95 \% \mathrm{CI}, 0.08-0.71)$. This meta-analysis included a total of 193 type 1 diabetic patients in 3 different studies, with duration of diabetes in excess of 20 years, and follow-up after transplant for a median of 5-7 years $(46,47)$.

Data from pancreas transplants compared with nontransplanted diabetic controls shows similar evidence (46). Isolated pancreas transplantation $(\mathrm{RR}=0.16$; 95\% CI, 0.05-0.49) and combined pancreas-kidney transplantation (RR 0.21; 95\% CI, 0.09-0.52) were associated with reduced risk of DR progression compared with controls. $(46,48)$. These studies were carried out in 159 patients in total, but with a shorter median follow up, only 18-29 months. Mean diabetes duration was also greater than 20 years prior to transplant.

These beneficial effects after transplant cannot prove, but are consistent with, a role for beta cell secretion products over exogenous insulin in preventing or reversing progression of DR. More data are needed to demonstrate a firm role for endogenous insulin that also take into account the possible influence of other factors, such as glycemia, islet isolation methods, and immunosuppressive regimens. Notwithstanding these factors, the transplant data also support a role for endogenous insulin in DR protection.

There are, however, two important caveats that need to be mentioned: (a) results of short-term follow up studies of pancreas transplant and DR, and (b) a putative role for C-peptide in DR.

The early period after pancreas transplantation is a complex setting to assess the effect of beta cell replacement because two dominant processes occur simultaneously: successful reinitiation of beta cell function and endogenous insulin secretion; and rapid decline in glycemia. These two processes have opposite effects on the retina. In the case of rapid glucose control, worsening is a well-documented phenomenon $(49,50)$. After pancreas transplant, similar deterioration has been 
demonstrated in short-term trials, conceivably explaining DR progression within a few months of transplant $(51,52)$, as opposed to the longer follow-up trials mentioned above (46-48). It is therefore essential to examine the effect of pancreas transplant early and also after long-term follow-up.

Since endogenous beta cell function consists of both insulin and C-peptide secretion, it should be noted that there are data that support the concept that there is a direct biological effect of C-peptide on the microvasculature (44). C-peptide has been shown to decrease glucose-induced apoptosis of endothelial cells, prevent oxidative stress through an antioxidant role, reducing RAC-1 translocation to the membrane and $\mathrm{NAD}(\mathrm{P}) \mathrm{H}$ oxidase activation (53). Thus it is conceivable that the role described here for residual endogenous beta cell secretion may be mediated by $\mathrm{C}$-peptide action as well as, or instead of, insulin.

\section{DISCUSSION AND CONCLUSIONS}

In this perspective, we have assembled information available to support the concept that endogenous insulin secretion plays an important role in the prevention of DR, that is not achieved by exogenous insulin alone. The presence of insulin receptors in the retina sets the stage for an active biological role for insulin in the eye. Although exogenous insulin may also interact with these insulin receptors, evidence is presented that supports a role for endogenous insulin in protecting against DR in both type 1 and type 2 diabetes. Insulin treatment in type 2 diabetes, reflecting endogenous insulin insufficiency, is an important risk factor for DR. Cross-sectional and prospective clinical studies accentuate the role of endogenous insulin deficiency in the development of DR in both type 1 and type 2 diabetes. Replacing beta cells by islet cell transplants prevents progression of $\mathrm{DR}$ in people with type 1 diabetes, direct support for the importance of endogenous insulin secretion to prevent DR. A caveat for many of these studies is that insulin deficiency is commonly associated with poor glycemic control, which is itself a risk factor for DR. However many of the studies quoted in this perspective either

\section{REFERENCES}

1. Naeser P. Insulin Receptors in Human Ocular Tissues: Immunohistochemical Demonstration in Normal and Diabetic Eyes. Uppsala J Med Sci (1997) 102:35-40. doi: 10.3109/03009739709178930

2. Rains JL, Jain SK. Oxidative Stress, Insulin Signaling, and Diabetes. Free Radical Biol Med (2011) 50:567-75. doi: 10.1016/j.freeradbiomed.2010.12.006

3. Early Treatment Diabetic Retinopathy Study Research Group. Grading Diabetic Retinopathy From Stereoscopic Color Fundus Photographs-an Extension of the Modified Airlie House Classification. ETDRETDRS Report Number 10. Ophthalmology (1991) 98:786-806. doi: 10.1016/S0161-6420(13) 38012-9

4. Simó R, Stitt AW, Gardner TW. Neurodegeneration in Diabetic Retinopathy: Does It Really Matter? Diabetologia (2018) 61:1902-12. doi: 10.1007/s00125018-4692-1

5. Rong X, Ji Y, Zhu X, Yang J, Qian D, Mo X, et al. Neuroprotective Effect of Insulin-Loaded Chitosan Nanoparticles/PLGA-PEG-PLGA Hydrogel on Diabetic Retinopathy in Rats. Int J Nanomed (2019) 14:45-55. doi: 10.2147/ IJN.S184574 adjusted for glycemic control or demonstrated a C-peptide benefit even in tightly controlled patients; thus insulin deficiency remained an independent risk factor for DR.

The accumulation of data presented here strongly suggests that when beta cell replacement is more readily available, an additional indication for its use could be to protect the retina in patients with type 1 diabetes and even insulin deficient type 2 diabetes, an even larger population who could potentially benefit from this approach.

Future prospective clinical studies of beta cell replacement should include progression of DR as an essential outcome measure.

\section{DATA AVAILABILITY STATEMENT}

The original contributions presented in the study are included in the article/supplementary material. Further inquiries can be directed to the corresponding author.

\section{AUTHOR CONTRIBUTIONS}

The author confirms being the sole contributor of this work and has approved it for publication.

\section{ACKNOWLEDGMENTS}

The author is indebted to the support and outstanding work of Pauline Genter MS RD in development of our understanding of DR and its relationship to diminished beta cell function. Grant support for work included in this Perspective: the National Institutes of Health (EY014684) and the Clinical and Translational Science Institute (CTSI), supported by the National Center for Advancing Translational Sciences, CTSI grant UL1TR000124, and the Norris Foundation. EI also has research funding from Eyenuk, Inc not related to this study.

6. Tarchick MJ, Cutler AH, Trobenter TD, Kozlowski MR, Makowski ER, Holoman N, et al. Endogenous Insulin Signaling in the RPE Contributes to the Maintenance of Rod Photoreceptor Function in Diabetes. Exp Eye Res (2019) 180:63-74. doi: 10.1016/j.exer.2018.11.020

7. De Pablo F, Hernandez-Sanchez C, de la Rosa EJ. The Prohormone Proinsulin as a Neuroprotective Factor: Past History and Future Prospects. Front Mol Neurosci (2018) 11:426. doi: 10.3389/fnmol.2018.00426

8. Barber AJ, Nakamura M, Wolpert EB, Reiter CE, Seigel GM, Antonetti DA, et al. Insulin Rescues Retinal Neurons From Apoptosis by a Phosphatidylinositol 3-Kinase/Akt-Mediated Mechanism That Reduces the Activation of Caspase-3. J Biol Chem (2001) 276:32814-21. doi: 10.1074/ jbc.M104738200

9. Meyer-Franke A, Kaplan MR, Pfrieger FW, Barres BA. Characterization of the Signaling Interactions That Promote the Survival and Growth of Developing Retinal Ganglion Cells in Culture. Neuron (1995) 15:805-19. doi: 10.1016/ 0896-6273(95)90172-8

10. Wu X, Reiter CE, Antonetti DA, Kimball SR, Jefferson LS, Gardner TW. Insulin Promotes Rat Retinal Neuronal Cell Survival in a P70s6k-Dependent Manner. J Biol Chem (2004) 279:9167-75. doi: 10.1074/jbc.M312397200 
11. Rajala A, Tanito M, Le YZ, Kahn CR, Rajala RV. Loss of Neuroprotective Survival Signal in Mice Lacking Insulin Receptor Gene in Rod Photoreceptor Cells. J Biol Chem (2008) 283:19781-92. doi: 10.1074/jbc.M802374200

12. Ivanovic I, Anderson RE, Le YZ, Fliesler SJ, Sherry DM, Rajala RV. Deletion of the P85alpha Regulatory Subunit of Phosphoinositide 3-Kinase in Cone Photoreceptor Cells Results in Cone Photoreceptor Degeneration. Investig Ophthalmol Vis Sci (2011) 52:3775-83. doi: 10.1167/iovs.10-7139

13. Punzo C, Kornacker K, Cepko CL. Stimulation of the Insulin/mTOR Pathway Delays Cone Death in a Mouse Model of Retinitis Pigmentosa. Nat Neurosci (2009) 12:44-52. doi: 10.1038/nn.2234

14. Rajala RV, Kanan Y, Anderson RE. Photoreceptor Neuroprotection: Regulation of Akt Activation Through Serine/Threonine Phosphatases, PHLPP and PHLPPL. Adv Exp Med Biol (2016) 854:419-24. doi: 10.1007/ 978-3-319-17121-0_55

15. Yu X, Rajala RV, McGinnis JF, Li F, Anderson RE, Yan X, et al. Involvement of Insulin/Phosphoinositide 3-Kinase/Akt Signal Pathway in 17 BetaestradiolMediated Neuroprotection. J Biol Chem (2004) 279:13086-94. doi: 10.1074/ jbc.M313283200

16. Rajala A, Dighe R, Agbaga MP, Anderson RE, Rajala RV. Insulin Receptor Signaling in Cones. J Biol Chem (2013) 288:19503-15. doi: 10.1074/ jbc.M113.469064

17. The Diabetes Control and Complications Trial/Epidemiology of Diabetes Interventions and Complications Research Group. Retinopathy and Nephropathy in Patients With Type 1 Diabetes Four Years After a Trial of Intensive Therapy. N Engl J Med (2000) 342:381-9. doi: 10.1056/ NEJM200002103420603

18. UK Prospective Diabetes Study (UKPDS) Group. Intensive Blood-Glucose Control With Sulphonylureas or Insulin Compared With Conventional Treatment and Risk of Complications in Patients With Type 2 Diabetes (UKPDS 33). Lancet (1998) 352:837-53. doi: 10.1016/S0140-6736(98)07019-6

19. Hirsch IB, Brownlee M. Beyond Hemoglobin Alc-Need for Additional Markers of Risk for Diabetic Microvascular Complications. JAMA (2010) 303:2291-2. doi: 10.1001/jama.2010.785

20. Duckworth W, Abraira C, Moritz T, Reda D, Emanuele N, Reaven PD, et al. Glucose Control and Vascular Complications in Veterans With Type 2 Diabetes. N Engl J Med (2009) 360:129-39. doi: 10.1056/NEJMoa0808431

21. Patel A, MacMahon S, Chalmers J, Neal B, Billot L, Woodward M, et al. Intensive Blood Glucose Control and Vascular Outcomes in Patients With Type 2 Diabetes. N Engl J Med (2008) 358:2560-72. doi: 10.1056/ NEJMoa0802987

22. Steffes MW, Sibley S, Jackson M, Thomas W. Beta-Cell Function and the Development of Diabetes-Related Complications in the Diabetes Control and Complications Trial. Diabetes Care (2003) 26:832-6. doi: 10.2337/ diacare.26.3.832

23. Gubitosi-Klug RA, Braffett BA, Hitt S, Arends V, Uschner D, Jones K, et al. For the DCCT/EDIC Research Group. Residual $\beta$ Cell Function in Long-Term Type 1 Diabetes Associates With Reduced Incidence of Hypoglycemia. J Clin Invest (2021) 131:e143011. doi: 10.1172/JCI143011

24. Suzuki K, Watanabe K, Motegi T, Kajinuma H. High Prevalence of Proliferative Retinopathy in Diabetic Patients With Low Pancreatic B-Cell Capacity. Diabetes Res Clin Pract (1989) 6:45-52. doi: 10.1016/0168-8227(89) 90056-9

25. Bo S, Gentile L, Castiglione A, Prandi V, Canil S, Ghigo E, et al. C-Peptide and the Risk for Incident Complications and Mortality in Type 2 Diabetic Patients: A Retrospective Cohort Study After a 14-Year Follow-Up. Eur J Endocrinol (2012) 167:173-80. doi: 10.1530/EJE-12-0085

26. Yoon HJ, Cho YZ, Kim JY, Kim BJ, Park KY, Koh GP, et al. Correlations Between Glucagon Stimulated C-Peptide Levels and Microvascular Complications in Type 2 Diabetes Patients. Diabetes Metab J (2012) 36:379-87. doi: 10.4093/dmj.2012.36.5.379

27. Kim BY, Jung CH, Mok JO, et al. Association Between Serum C-Peptide Levels and Chronic Microvascular Complications in Korean Type 2 Diabetic Patients. Acta Diabetol (2012) 49:9-15. doi: 10.1007/s00592-0100249-6

28. Snehalatha C, Mohan R, Mohan V, Ramachandran A, Viswanathan M. Pancreatic B-Cell Function in Relation to Diabetic Retinopathy in Asian Indian NIDDM Patients. Acta Diabetol Lat (1988) 25:95-100. doi: 10.1007/ BF02581372
29. Sari R, Balci MK. Relationship Between C Peptide and Chronic Complications in Type-2 Diabetes Mellitus. J Natl Med Assoc (2005) 97:1113-8.

30. Boz M, Scheen AJ, Gerard PL, Castillo MJ, Lefebvre PJ. Retinopathy, But Not Neuropathy, Is Influenced by the Level of Residual Endogenous Insulin Secretion in Type 2 Diabetes. Diabetes Metab (1995) 21:353-9.

31. Kuo JZ, Guo X, Klein R, et al. Association of Fasting Insulin and C Peptide With Diabetic Retinopathy in Latinos With Type 2 Diabetes. BMJ Open Diabetes Res Care (2014) 2(1):e000027. doi: 10.1136/bmjdrc-2014-000027

32. Shiraiwa T, Kaneto H, Miyatsuka T, Kato K, Yamamoto K, Kawashima A, et al. Post-Prandial Hyperglycemia Is an Important Predictor of the Incidence of Diabetic Microangiopathy in Japanese Type 2 Diabetic Patients. Biochem Biophys Res Commun (2005) 336:339-45. doi: 10.1016/j.bbrc.2005.08.158

33. Tudor SM, Hamman RF, Baron A, Johnson DW, Shetterly SM. Incidence and Progression of Diabetic Retinopathy in Hispanics and Non-Hispanic Whites With Type 2 Diabetes. San Luis Valley Diabetes Study, Colorado. Diabetes Care (1998) 21:53-61. doi: 10.2337/diacare.21.1.53

34. Jones CD, Greenwood RH, Misra A, Bachmann MO. Incidence and Progression of Diabetic Retinopathy During 17 Years of a Population-Based Screening Program in England. Diabetes Care (2012) 35:592-6. doi: 10.2337/ dc11-0943

35. Thomas RL, Dunstan F, Luzio SD, Chowdury S, Hale SL, North RV, et al. Incidence of Diabetic Retinopathy in People With Type 2 Diabetes Mellitus Attending the Diabetic Retinopathy Screening Service for Wales: Retrospective Analysis. BMJ (2012) 344:e874. doi: 10.1136/bmj.e874

36. Pearson ER. Type 2 Diabetes: A Multifaceted Disease. Diabetologia (2019) 62:1107-12. doi: 10.1007/s00125-019-4909-y

37. Wong TY, Sabanayagam C. The War on Diabetic Retinopathy: Where Are We Now? Asia Pac J Ophthalmol (Phila) (2019) 8:448-56. doi: 10.1097/ APO.0000000000000267

38. Yoshihara E, O'Connor C, Gasser E, Wei Z, Oh TG, Tseng TW, et al. Immune-Evasive Human Islet-Like Organoids Ameliorate Diabetes. Nature (2020) 586:606-11. doi: 10.1038/s41586-020-263-z

39. Ahlqvist E, Storm P, Käräjämäki A, Martinelli M, Dorkhan M, Carlsson A, et al. Novel Subgroups of Adult-Onset Diabetes and Their Association With Outcomes: A Data-Driven Cluster Analysis of Six Variables. Lancet Diabetes Endocrinol (2018) 6:361-69. doi: 10.1016/S2213-8587(18)30051-2

40. Ahlqvist E, Prasad RB, Groop L. Subtypes of Type 2 Diabetes Determined From Clinical Parameters. Diabetes (2020) 69:2086-93. doi: 10.2337/dbi20-0001

41. Zou X, Zhou X, Zhu Z, Ji L. Novel Subgroups of Patients With Adult-Onset Diabetes in Chinese and US Populations. Lancet Diabetes Endocrinol (2019) 7:9-11. doi: 10.1016/S2213-8587(18)30316-4

42. Tanabe H, Saito H, Kudo A, Machii N, Hirai H, Maimaituxun G, et al. Factors Associated With Risk of Diabetic Complications in Novel Cluster-Based Diabetes Subgroups: A Japanese Retrospective Cohort Study. J Clin Med (2020) 9:2083. doi: $10.3390 / \mathrm{jcm} 9072083$

43. Anjana RM, Baskar V, Nair ATN, Jebarani S, Siddiqui MK, Pradeepa R, et al. Novel Subgroups of Type 2 Diabetes and Their Association With Microvascular Outcomes in an Asian Indian Population: A Data-Driven Cluster Analysis: The INSPIRED Study. BMJ Open Diabetes Res Care (2020) 8:e001506. doi: 10.1136/bmjdrc-2020-001506

44. Bhatt MP, Lim Y-C, Ha KS. C-Peptide Replacement Therapy as an Emerging Strategy for Preventing Diabetic Vasculopathy. Cardiovasc Res (2014) 104:234-44. doi: 10.1093/cvr/cvu211

45. Azad N, Agrawal L, Emanuele NV, Klein R, Bahn GD, Reaven P. VADT Study Group. Association of Blood Glucose Control and Pancreatic Reserve With Diabetic Retinopathy in the Veterans Affairs Diabetes Trial (VADT). Diabetologia (2014) 57:1124-31. doi: 10.1007/s00125-014-3199-7

46. Virk SA, Donaghue KC, Wong TY, Craig MA. Interventions for Diabetic Retinopathy in Type 1 Diabetes: Systematic Review and Meta-Analysis. Am J Ophthalmol (2015) 160:1055-64. doi: 10.1016/j.ajo.2015.07.024

47. Thompson DM, Begg IS, Harris C, Ao Z, Fung RM, Keown P, et al. Reduced Progression of Diabetic Retinopathy After Islet Cell Transplantation Compared With Intensive Medical Therapy. Transplantation (2008) 85:1400-05. doi: 10.1097/TP.0b013e318172ca07

48. Giannarelli R, Coppelli A, Sartini MS, Del Chiaro M, Vistoli F, Rizzo G, et al. Pancreas Transplant Alone has Beneficial Effects on Retinopathy in Type 1 Diabetic Patients. Diabetologia (2006) 49:2977-82. doi: 10.1007/s00125-0060463-5 
49. Diabetes Control and Complications Trial Research Group, Nathan DM, Genuth S, Lachin J, Cleary P, Crofford O, Davis M, et al. The Effect of Intensive Treatment of Diabetes on the Development and Progression of Long-Term Complications in Insulin-Dependent Diabetes Mellitus. N Engl J Med (1993) 329:977-86. doi: 10.1056/NEJM199309303291401

50. Shurter A, Genter P, Ouyang D, Ipp E. Euglycemic Progression: Worsening of Diabetic Retinopathy in Poorly Controlled Type 2 Diabetes in Minorities. Diabetes Res Clin Pract (2013) 100:362-67. doi: 10.1016/j.diabres.2013.03.018

51. Tsai F-Y, Lau L-I, Li AF, Chen SJ, Wang SE, Lee FL, et al. Acute Macular Edema and Peripapillary Soft Exudate After Pancreas Transplantation With Accelerated Progression of Diabetic Retinopathy. J Chin Med Assoc (2017) 80:319-25. doi: 10.1016/j.jcma.2017.01.004

52. Voglová B, Hladíková Z, Nemétová L, Zahradnická M, Kesslerová K, Sosna T, et al. Early Worsening of Diabetic Retinopathy After Simultaneous Pancreas and Kidney Transplantation-Myth or Reality? Am J Transplant (2020) 20:2832-41. doi: 10.1111/ajt.15924

53. Cifarelli V, Geng X, Styche A, Lakomy R, Trucco M, Luppi P. C-Peptide Reduces High-Glucose-Induced Apoptosis of Endothelial Cells and Decreases NAD(P)H-Oxidase Reactive Oxygen Species Generation in Human Aortic
Endothelial Cells. Diabetologia (2011) 54:2702-12. doi: 10.1007/s00125-0112251-0

Conflict of Interest: The author declare that the research was conducted in the absence of any commercial or financial relationships that could be construed as a potential conflict of interest.

Publisher's Note: All claims expressed in this article are solely those of the authors and do not necessarily represent those of their affiliated organizations, or those of the publisher, the editors and the reviewers. Any product that may be evaluated in this article, or claim that may be made by its manufacturer, is not guaranteed or endorsed by the publisher.

Copyright (c) 2021 Ipp. This is an open-access article distributed under the terms of the Creative Commons Attribution License (CC BY). The use, distribution or reproduction in other forums is permitted, provided the original author(s) and the copyright owner(s) are credited and that the original publication in this journal is cited, in accordance with accepted academic practice. No use, distribution or reproduction is permitted which does not comply with these terms. 